\title{
Folded-Path Self-Pumped Wavelength Converter Based on Four-Wave Mixing in a Semiconductor Optical Amplifier
}

\author{
Guido Hunziker, Roberto Paiella, Student Member, IEEE, Mehrdad Ziari, \\ Atul Mathur, and Kerry J. Vahala, Member, IEEE
}

\begin{abstract}
A four-wave mixing wavelength converter with no external pump laser and very low input signal power requirements is characterized. The wavelength conversion occurs inside a high-reflection/antireflection coated semiconductor optical amplifier pigtailed with a fiber Bragg grating. The pump signal is provided by the lasing mode at the Bragg wavelength. A $1-\mathrm{mW}$ optical signal modulated at $2.5 \mathrm{~Gb} / \mathrm{s}$ is converted over $9 \mathrm{~nm}$ with error rates below $10^{-9}$.
\end{abstract}

Index Terms-Communication systems, optical frequency conversion, optical mixers, optical propagation in nonlinear media, optical saturation, semiconductor lasers, semiconductor optical amplifiers, wavelength-division multiplexing.

A LL-OPTICAL wavelength converters and signalprocessing devices are likely to be important components in future wavelength-division multiplexed (WDM) networks. To date, technologies based on cross-gain modulation (XGM) and cross-phase modulation (XPM) of optical signals are more technologically mature as compared to wave-mixing based techniques [1]. However, the advantages of wave-mixing techniques include strict bit-rate and modulation format transparency [2], multichannel operation [3] as well as the possibility of other processing functions such as optical phase conjugation [4] and optical logic gates [5]. In addition, large spectral and dynamic range [6], cascadability [7], and polarization independence [8]-[10] are all achievable, but more difficult to implement than with XGM or XPM. To be technologically competitive, wave-mixing frequency converters need good conversion efficiency, high signalto-noise ratio (SNR) on the converted signal, and reduced complexity. In this letter, we present a promising four-wave mixing (FWM) technique that addresses these three criteria simultaneously.

A straightforward strategy to improve the FWM performance in semiconductor optical amplifiers (SOA's) is to increase the amplifier gain-length product and/or the pump power. This has led to good converter performance, using high-power erbium-doped fiber amplifiers (EDFA's) and long

Manuscript received May 16, 1997; revised June 19, 1997. This work was supported by Advanced Research Projects Agency under Grant DAAL 01-94k-03430, by the National Science Foundation under Grant ECS 9412862, and by the NORTHROP Corporation.

G. Hunziker, R. Paiella, and K. J. Vahala are with the Department of Applied Physics, California Institute of Technology, Pasadena, CA 91125 USA.

M. Ziari and A. Mathur are with SDL Inc., San Jose, CA 95134 USA.

Publisher Item Identifier S 1041-1135(97)07188-7.

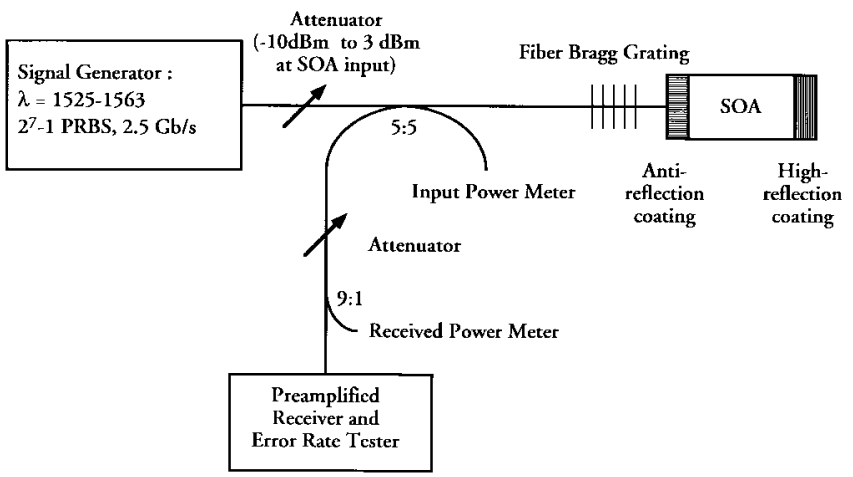

Fig. 1. Schematic of the FWM wavelength conversion experiment.

SOA's [6], [11] or high-power semiconductor lasers as frequency converters [10], [12]. The self-pumped (lasing) and folded-path configuration proposed in this letter is a candidate to implement the gain-length optimization strategy. Folding the modulated signal path by reflection off one of the cavity mirrors doubles the interaction length, while the lasing action maintains the gain (and hence, the nonlinearity) clamped at the threshold value (as opposed to external pumping where the gain is saturated by the total input power). Furthermore, the fiber Bragg grating used at the other end of the lasing cavity can, in principle, be optimized to achieve high internal lasing power and good pump suppression at the output. Although the pump is not tunable in the present configuration, a similar scheme using a tunable DBR mirror instead of a Bragg grating could be envisioned.

The converter is a small external cavity semiconductor laser, where the feedback is provided on one end of the semiconductor by a high-reflection coating, and on the other end (antireflection coated) by a pigtailed fiber Bragg grating (see Fig. 1). The cavity length and reflectivity profiles are optimized for single-mode laser oscillation. The active region is 500- $\mu \mathrm{m}$-long, temperature controlled, and biased at 170 $\mathrm{mA}$. The lasing threshold is $32 \mathrm{~mA}$ and the pump output power at the operating point is $+7.5 \mathrm{dBm}$. Modulated input signals (with wavelengths outside of the Bragg reflection bandwidth) are injected into the cavity through the Bragg grating using a bidirectional coupler. The converted signal is collected from the other input arm of the coupler. A tunable external-cavity semiconductor laser is externally modulated at $2.5 \mathrm{~Gb} / \mathrm{s}$ by a pseudorandom bit stream $\left(2^{7}-1\right.$ PRBS, no 


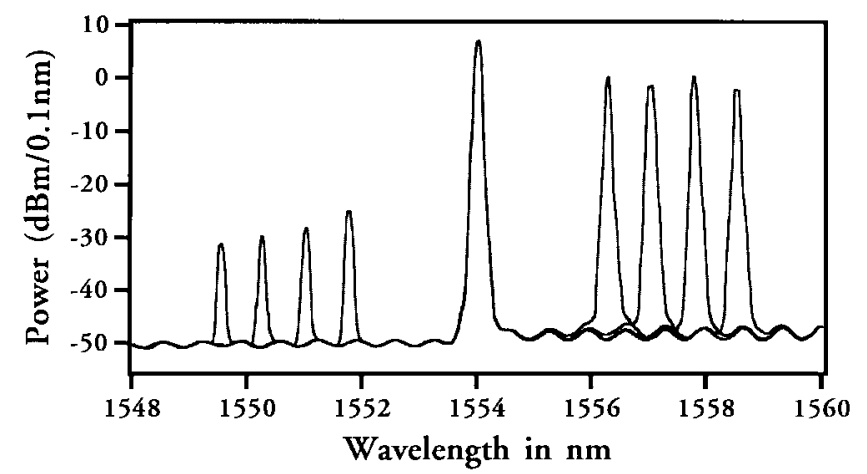

(a)

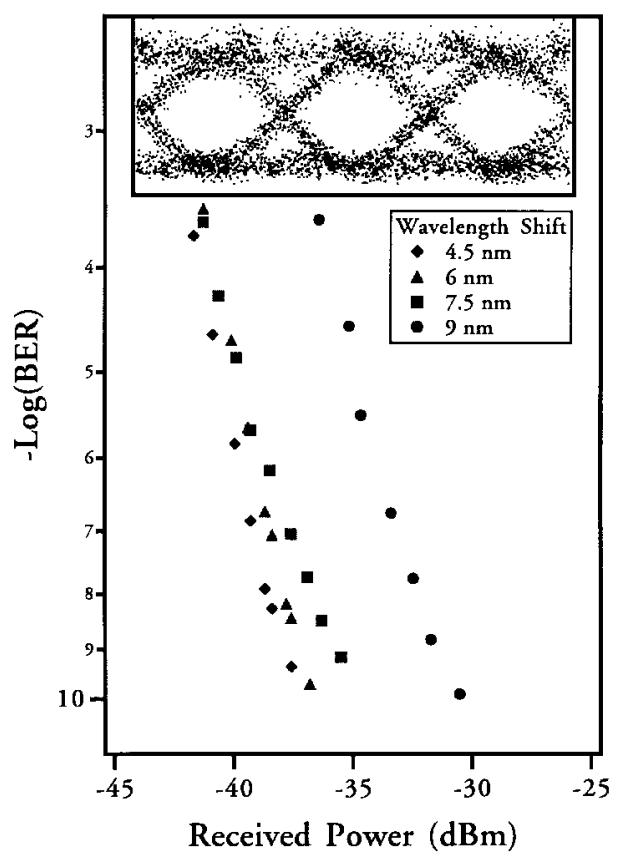

(b)

Fig. 2. (a) Superimposed optical spectra measured at the output port of the converter. The wavelength shifts are $4.5,6,7.5$, and $9 \mathrm{~nm}$. (b) Top: eye diagram of the error-free converted signal for a $4.5-\mathrm{nm}$ shift with $1-\mathrm{mW}$ input signal power to the SOA (after the bidirectional coupler). Bottom: BER curves versus received power for the wavelength shifts in (a).

significant wordlength dependence was observed), generated in a bit-error-rate (BER) tester (HP 70800 Series). The signal is amplified in an EDFA and filtered by a 1-nm bandpass filter to reduce the amplified spontaneous emission (ASE) noise in the converted signal band. An attenuator is used in order to measure the input signal dynamic range of the converter. The converted signal is attenuated and measured before going to the preamplified receiver at the detection stage. The optically preamplified receiver consists of a 1-nm bandpass filter, an EDFA, and another 1-nm bandpass filter in series. The overall small-signal gain is about $29 \mathrm{~dB}$. Optical to electrical signal conversion is done in an HP 8347 Lightwave converter followed by an HP 11982 RF-frequency amplifier and a $2.5-\mathrm{Gb} / \mathrm{s}$ Bessel-Thomson filter.

Spectra of the light at the output of the device are superimposed in Fig. 2(a) for different wavelength shifts ranging from 4.5 to $9 \mathrm{~nm}$. Fig. 2(b) shows an eye diagram of the

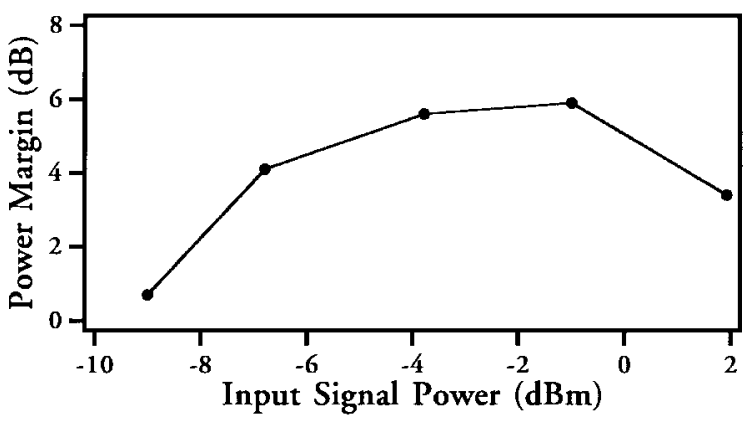

Fig. 3. Power margin (admissible converted signal attenuation before the preamplifier for error rates below $10^{-9}$ ) versus input signal power to the converter.

error-free converted signal for $1-\mathrm{mW}$ signal input and a 4.5$\mathrm{nm}$ wavelength shift. The lower part of the figure shows the measured BER versus received power for the shifts in Fig. 2(a). The large received power required for the highest $(9 \mathrm{~nm})$ wavelength shift stems from the reduced conversion efficiency for larger shifts.

Finally, in Fig. 3, we show the power margin on the converted signal for various input signal power levels. The power margin is defined as the amount of attenuation of the converted signal necessary to degrade the error rates to $10^{-9}$. The wavelength shift was $4.5 \mathrm{~nm}$ in this case. For the low-input power regime, the power margin drops because of insufficient optical SNR's on the converted signals, while on the highpower end, the margin is reduced because the laser oscillation is partially quenched and, therefore, the pump power is lower. Notice that the nature of this degradation at high input power is expected to be fundamentally different from that of a nonlasing wavelength converter [13]. In particular, the value of the gain is clamped at the threshold value in the self-pumped device whereas it experiences saturation in a conventional nonlasing converter. This distinction should reduce, for example, parasitic chirp in the current device [14].

The measured conversion efficiency of this device is comparable to that measured in a similar structure but twice as long and in the usual single-pass FWM configuration [6]. The optical SNR is currently limiting the performance of this converter. By using high-reflection Bragg gratings, the threshold current could be reduced and that would further lower the noise floor of the converter. High Bragg reflectivity would simultaneously suppress the pump wave at the output of the device, a desirable feature in FWM converters. Furthermore, improvements associated with the use of longer SOA's [11] are expected to be even more dramatic in the lasing foldedpath configuration than in a single-pass configuration, since the length of the device is used twice in this case. We also note that by using an optical circulator instead of a bidirectional coupler in this design a further increase by $3 \mathrm{~dB}$ in the power level of the converted signal would result.

In conclusion, we have presented a FWM wavelength converter which eliminates the need for a separate pump laser, high-power optical signal amplifiers, and ASE prefiltering normally used in single-pass converters [6]. Required input signal power levels are comparable to commonly used levels in XGM or XPM. The design presented here, combined with 
highly reflecting Bragg gratings and longer amplifying sections is expected to provide an improvement over conventional mixer-based converters.

\section{ACKNOWLEDGMENT}

The authors would like to thank Dr. T. Schrans and Dr. N. Kwong for loaning an electronic amplifier and Dr. D. Geraghty and Dr. R. Lee for their help and advice.

\section{REFERENCES}

[1] T. Durhuus, B. Mikkelsen, and C. Joergensen, "All-optical wavelength conversion by semiconductor optical amplifiers," J. Lightwave Technol., vol. 14, pp. 942-954, 1996.

[2] J. Zhou, N. Park, J. W. Dawson, K. J. Vahala, M. A. Newkirk, and B I. Miller, "Efficiency of broadband four-wave mixing wavelength conversion using semiconductor traveling-wave amplifiers," IEEE Photon. Technol. Lett., vol. 6, pp. 50-52, 1994.

[3] J. P. R. Lacey, S. J. Madden, M. A. Summerfield, R. S. Tucker, and A. I. Faris, "Four-channel WDM optical phase conjugator using four-wave mixing in a single semiconductor optical amplifier," Electron. Lett., vol. 31, pp. 743-744, 1995.

[4] M. C. Tatham, X. Gu, L. D. Westbrook, G. Sherlock, and D. M. Spirit, "Transmission of $10 \mathrm{Gbit} / \mathrm{s}$ directly modulated DFB signals over 200 $\mathrm{km}$ standard fiber using mid-span spectral inversion," Electron. Lett., vol. 30, pp. 1335-1336, 1994.

[5] J. Vahala, R. Paiella, and G. Hunziker, "Ultrafast WDM logic," J. Select. Topics Quantum Electron., vol. 3, pp. 698-701, Apr. 1997.

[6] D. F. Geraghty, R. B. Lee, K. J. Vahala, M. Verdiell, M. Ziari, and A. Mathur, "Wavelength conversions up to $18 \mathrm{~nm}$ at $10 \mathrm{~Gb} / \mathrm{s}$ by four-wave- mixing in a semiconductor optical amplifier," IEEE Photon. Technol. Lett., vol. 9, pp. 452-454, 1997.

[7] R. B. Lee, D. F. Geraghty, K. J. Vahala, M. Verdiell, M. Ziari, and A. Mathur, "Cascaded wavelength conversion by four-wave-mixing in a strained semiconductor optical amplifier at $10 \mathrm{~Gb} / \mathrm{s}$," IEEE Photon. Technol. Lett., vol. 9, pp. 752-754, June 1997.

[8] G. Hunziker, R. Paiella, D. F. Geraghty, K. J. Vahala, and U. Koren, "Polarization-independent wavelength conversion at $2.5 \mathrm{Gbit} / \mathrm{sec}$ by dual-pump four-wave mixing in a strained semiconductor optical amplifier," IEEE Photon. Technol. Lett., vol. 8, pp. 1633-1635, 1996.

[9] P. Cortes, M. Chbat, S. Artigaud, J.-1. Beylat, and J. Chesnoy, "Below $0.3 \mathrm{~dB}$ polarization penalty in $10 \mathrm{~Gb} / \mathrm{s}$ directly modulated DFB signal over $160 \mathrm{~km}$ using mid-span spectral inversion in a semiconductor optical amplifier," in Proc. ECOC'95, Brussels, Belgium, 1995, pp. 271-274, paper Tu.B.2.3

[10] S. Watanabe, H. Kuwastuka, S. Takeda, and H. Ishikawa, "Polarizationinsensitive wavelength conversion and phase conjugation using bidirectional forward four-wave mixing in a lasing DFB-LD," Electron. Lett., vol. 33, pp. 316-317, 1997.

[11] F. Martelli, A. Mecozzi, A. Dottavi, S. Scotti, and P. Spano, "Noise in wavelength conversion using 4 wave-mixing in semiconductor optical amplifiers," Appl. Phys. Lett., vol. 70, pp. 306-308, 1997.

[12] S. Murata, A. Tomita, J. Shimizu, and A. Suzuki, "THz opticalfrequency conversion of $1 \mathrm{~Gb} / \mathrm{s}$ signals using highly nondegenerate FWM in an InGaAsP semiconductor laser," IEEE Photon. Technol. Lett., vol. 3, pp. 1021-1023, 1991.

[13] M. Summerfield and R. Tucker, "Optimization of pump and signal powers for wavelength converters based on FWM in semiconductor optical amplifiers," IEEE Photon. Technol. Lett., vol. 8, pp. 1316-1318, 1996.

[14] D. Geraghty, R. Lee, K. Vahala, M. Verdiell, M. Ziari, and A. Mathur, "Time resolved spectral analysis of phase conjugation by four-wave mixing in semiconductor optical amplifiers," presented at CLEO Conf. Proc., Baltimore, MD, 1997, paper CTuH6. 